\title{
Why We Should Stop Using the Kogut and Singh Index
}

\author{
Palitha Konara ${ }^{1} \cdot$ Alexander Mohr $^{2}$ (D)
}

Received: 5 March 2018 / Revised: 16 February 2019 / Accepted: 25 February 2019 / Published online: 15 April 2019 (c) Springer-Verlag GmbH Germany, part of Springer Nature 2019

\begin{abstract}
The Kogut and Singh (J Int Bus Stud 19(3):411-432, 1988) index is the most widely used construct to measure cultural distance in international business and management research. We show that this index is incorrectly specified and captures the squared cultural distance. This inaccuracy is problematic because it means that the empirical findings on the effects of cultural distance presented in different strands of international business research are likely to be misleading. We specify the correct form of the distance measure based on the Euclidean distance formula and demonstrate the implications of using the incorrectly specified Kogut and Singh (1988) index.
\end{abstract}

Keywords Cultural distance $\cdot$ Kogut-Singh Index $\cdot$ Euclidian distance

\section{Introduction}

The existence of differences between countries and regions in which firms do business is the conditio sine qua non for the discipline of international business. The concept of distance capturing the degree to which countries and regions differ lies at the core of international business and management research. Zaheer et al. (2012, p. 19) stress that international management is "essentially, [...] the management of distance". Depending on the type of difference studied, prior research has suggested various types of distance, although cultural distance, i.e., the extent to which countries differ in cultural values, remains the most widely used type of distance in international business (Beugelsdijk et al. 2018b; Shenkar et al. 2008; Tihanyi et al. 2005). Research into the effects of cultural distance exploded with the introduction by Kogut and Singh (1988) of an index to measure this distance. The Kogut-Singh Index (KSI) aggregates cultural differences along Hofstede (1980) four dimensions, i.e., power distance, uncertainty avoidance, individualism, and masculinity, into a

Alexander Mohr

alexander.mohr@wu.ac.at

1 University of Sussex Business School, University of Sussex, Falmer, Brighton, UK

2 Vienna University of Economics and Business, Vienna, Austria 


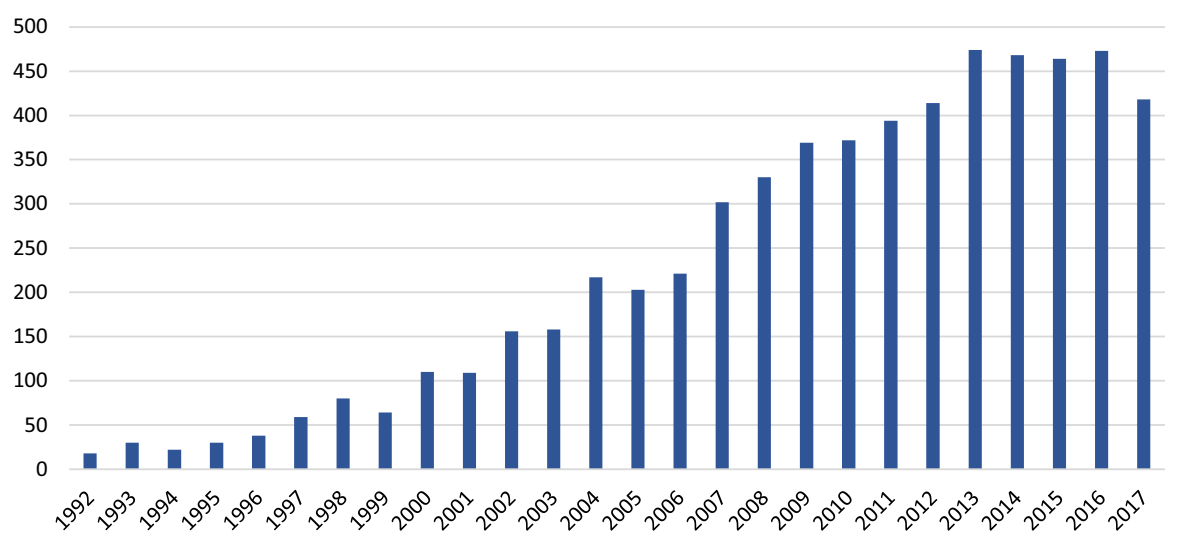

Fig. 1 Citations of Kogut and Singh (1988). Source: Google Scholar

single value. Kogut and Singh (1988) developed their index to explore the effect that cultural distance has on firms' choice of foreign market entry mode choice. They argued that increasing cultural distance between an investor's home country and the target market would lead to a greater preference for joint ventures or wholly-owned green-field ventures over acquisitions. Subsequently, the KSI has not only been employed in empirical studies of the choice of firms' foreign market entry, but also been used to capture cultural distance in empirical studies on a very wide range of IB topics at the macro- (e.g., Liu et al. 2018), meso- (e.g., Morosini et al. 1998; Shin et al. 2017; Yu and Maula 2016), and micro-level (e.g., Kraimer et al. 2012; Varela and Gatlin-Watts 2014).

To this day, the KSI remains the most widely used approach to measuring cultural distance (Beugelsdijk et al. 2018a; Cuypers et al. 2018). Studies reviewing research on cultural distance conclude that almost all of the reviewed studies used the KSI (Harzing and Pudelko 2016; Kirkman et al. 2006); thus, the original Kogut and Singh (1988) article is one of the most cited papers in the broader area of management, with more than 6000 citations to date (Beugelsdijk et al. 2018b; Harzing and Pudelko 2016). Further, the use of the KSI to measure (cultural) distance continues to increase (Harzing and Pudelko 2016) (see Fig. 1) despite repeated criticism levelled at the relevance of and the assumptions underlying the concept of cultural distance as such and/or the nature of the KSI to measure cultural and other types of distance (e.g., Berry et al. 2010; Harzing and Pudelko 2016; López-Duarte et al. 2016; Shenkar 2001; Tung and Verbeke 2010). Within the former group, scholars have questioned the implicit assumption of cultural distance being an absolute and symmetrical construct, as opposed to a relative and asymmetrical construct. Although these concerns are valid, we do not intend to engage in this generic debate on the usefulness of the notion of cultural distance, but instead aim to contribute to improving the measurement of cultural distance. Prior research in this latter group has suggested the use of perceptual data as opposed to relying on the data provided by Hofstede, Globe, or similar studies. Scholars have also raised the sensitivity of the index 
depending on the data that is used for its calculation (e.g., Beugelsdijk et al. 2018b) or problems associated with using the index on particular data (Gerschewski 2013).

We aim to contribute to improving the measurement of cultural distance in empirical research by showing that the KSI is a biased measure of cultural distance and fails to capture what it claims to capture. First, we demonstrate that the KSI differs fundamentally from the Euclidean distance. We show that because of a misspecification of the KSI, it corresponds to the squared Euclidean distance. As a result, empirical research that has employed the KSI has in fact used a squared function of cultural distance, leading to potentially misleading findings. We present the correct form of the Euclidean distance formula that we believe researchers should use in future studies. We also show that the KSI's deviation from the correct measure has important implications for the meaning of findings on the effects of cultural distance that were based on the KSI and that it is not possible to adjust or reinterpret the findings of prior empirical studies that employed the KSI.

The use of a squared function of cultural distance associated with the use of the KSI is likely a central explanation for the persistently inconclusive findings in research on the effects of cultural distance (Beugelsdijk et al. 2018b; Kirkman et al. 2006; Maseland et al. 2018). Rather than questioning the concept of cultural distance, we suggest that replacing the KSI with a correctly specified measure of cultural distance is likely to result in more consistent findings in future cultural distance research.

Replacing the KSI with a correct measure of distance is also important given that the KSI is increasingly being used for measuring cultural distance based on alternative cultural dimensions, such as the nine cultural dimensions reported by the GLOBE program (see for example, Chen et al. 2010; Hutzschenreuter and Voll 2008; Reus and Lamont 2009). Worryingly, the KSI is increasingly being used for capturing other types of distance. For example, recent research has used the KSI to measure regulatory distance (Salomon and $\mathrm{Wu} 2012$ ), governance and economic distance (Hutzschenreuter et al. 2014), differences in leadership (Koch et al. 2016) and business distance (Evans and Mavondo 2002). To prevent the problems characterizing the existing empirical research on the effects of cultural distance based on the KSI from spreading to these related areas of inquiry, we propose that researchers should opt for the correct measure of distance instead of using the KSI.

\section{The KSI as a Biased Estimator of (Cultural) Distance}

In their seminal article examining the effect of national culture on the choice of entry mode, Kogut and Singh (1988) introduced a composite index to measure the extent to which the country of the investing firm and the country of entry differ on the Hofstede's four national cultural dimensions of power distance, uncertainty avoidance, individualism, and masculinity. Based on this composite index, they showed that "the greater the cultural distance between the country of the investing firm and the country of entry, the more likely a firm will choose a joint venture or a wholly owned greenfield investment over an acquisition" (Kogut and Singh 1988, p. 414). Since then, their index "has become the field's standard-bearer, supplanting virtually 
all other modes of gauging cultural variations, including the prior concept of psychic distance" (Shenkar 2012, p. 12).

Kogut and Singh (1988) present the following formula to calculate the cultural distance between two countries. $\mathrm{KSI}_{\mathrm{ij}}$ is the cultural distance between country $\mathrm{i}$ and country j. $\mathrm{I}_{\mathrm{ki}}$ and $\mathrm{I}_{\mathrm{kj}}$ are the values of cultural dimension $\mathrm{k}(\mathrm{k}=1-4)$ for country $\mathrm{i}$ and country $\mathrm{j}$, respectively. $\mathrm{V}_{\mathrm{k}}$ is the variance of the cultural dimension $\mathrm{k}$.

$$
K S I_{i j}=\sum_{\mathrm{k}=1}^{4}\left(\frac{\left(\mathrm{I}_{\mathrm{ki}}-\mathrm{I}_{\mathrm{kj}}\right)^{2} / V_{k}}{4}\right)
$$

Kogut and Singh (1988, p. 422) do not provide a detailed explanation of this index apart from stating that "deviations were corrected for differences in the variances of each dimension and then arithmetically averaged" [emphasis added]. The standardization of the cultural dimensions scores prior to their summation is sensible to correct for the differences in the variances in each of the dimensions. However, the use of the arithmetic average of the standardized and squared differences creates a fundamental difference between the KSI and the Euclidean distance measure, despite some studies referring to the KSI as a 'Euclidean distance measure' (see for example, Beugelsdijk et al. 2018b; Cuypers et al. 2018; Tihanyi et al. 2005).

First, we look at the Euclidean distance measure. If $\mathrm{p}=\left(\mathrm{p}_{1}, \mathrm{p}_{2}, \ldots, \mathrm{p}_{\mathrm{n}}\right)$ and $\mathrm{q}=\left(\mathrm{q}_{1}\right.$, $\left.\mathrm{q}_{2}, \ldots, \mathrm{q}_{\mathrm{n}}\right)$ are two points in the Euclidean $\mathrm{n}$-space, then the distance $\left(\mathrm{d}_{\mathrm{pq}}\right)$ between $\mathrm{p}$ and $\mathrm{q}$ is given by the following (Anton and Rorres 2014, p. 145):

$$
\mathrm{d}_{p q}=\sqrt{\sum_{\mathrm{k}=1}^{n}\left(\mathrm{p}_{\mathrm{k}}-\mathrm{q}_{\mathrm{k}}\right)^{2}} .
$$

In the case of four cultural dimensions $(n=4)$, we obtain the following equation for calculating Euclidean (cultural) distance.

$$
\text { Euclidean (cultural) Distance } \text { Dij }=\sqrt{\sum_{\mathrm{k}=1}^{4}\left(\mathrm{I}_{\mathrm{ki}}-\mathrm{I}_{\mathrm{kj}}\right)^{2}} .
$$

A key difference between the KSI (Eq. 1) and the (non-standardized) Euclidean distance (Eq. 2) is that Kogut and Singh (1988) have adjusted (standardized) the deviations in each cultural dimension to address the differences in the variances across dimensions (by dividing each difference $p_{k}-q_{k}$ by the respective standard deviation $\mathrm{SD}_{\mathrm{k}}$ ). Similar to the Euclidean distance calculation, Kogut and Singh (1988) square these differences and sum them. Then, importantly, instead of taking the square root of the sum of the squared differences as per the Euclidean distance, Kogut and Singh (1988) divide this sum by four, i.e., they take the arithmetic average of the squared differences. By failing to take the square root of the sum of the squared differences, the KSI creates a second-degree (quadratic) function of distance. 
A small number of studies have used the (non-standardized) Euclidean distance (Eq. 3) to calculate cultural distance (see for example, Brouthers and Brouthers 2001; Manev and Stevenson 2001; Morosini et al. 1998). Although these studies have not standardized the individual cultural dimensions, Kogut and Singh (1988)'s standardization approach can be applied to the correct Euclidean distance formula by replacing the non-standardized cultural dimensions scores with the standardized cultural dimensions scores as below (Eq. 4a). In Eq. $4 \mathrm{a}, \mathrm{I}_{\mathrm{ki}}$ and $\mathrm{I}_{\mathrm{kj}}$ are the values of cultural dimension $\mathrm{k}(\mathrm{k}=1-4)$ for country $\mathrm{i}$ and country $\mathrm{j}$, respectively. $\mu_{\mathrm{k}}, \mathrm{SD}_{\mathrm{k}}$, and $\mathrm{V}_{\mathrm{k}}$ are the population mean, standard deviation and variance of cultural dimension $\mathrm{k}$, respectively.

$$
\text { Euclidean Distance }(\text { Standardized })_{i j}=\sqrt{\sum_{\mathrm{k}=1}^{4}\left(\left(\frac{\mathrm{I}_{\mathrm{ki}}-\mu_{\mathrm{k}}}{S D_{k}}\right)-\left(\frac{\mathrm{I}_{\mathrm{kj}}-\mu_{\mathrm{k}}}{S D_{k}}\right)\right)^{2}} \text {. }
$$

Equation $4 \mathrm{a}$ can be simplified into Eq. 4b, which is the standardized Euclidean distance formula that should be used to construct the composite cultural distance measure.

$$
\text { Euclidean Distance }(\text { Standardized })_{i j}=\sqrt{\sum_{\mathrm{k}=1}^{4} \frac{\left(\mathrm{I}_{\mathrm{ki}}-\mathrm{I}_{\mathrm{kj}}\right)^{2}}{V_{k}}}
$$

The correct formula for the standardized Euclidean distance differs markedly from the formula used in the KSI (Eq. 1). The formula used in the KSI (Eq. 1) and the correct formula (Eq. 4b) have the relationship shown in Eq. 5. Equation 5 shows that the KSI is a second-degree (quadratic) function of the correctly specified Euclidean distance. Thus, the KSI corresponds to a 'squared Euclidean distance', which is a special form of power distance that is discussed in applied mathematics (Deza and Deza 2016).

$$
K S I_{i j}=\frac{\left(\text { Euclidean Distance }(\text { Standardized })_{i j}\right)^{2}}{4} .
$$

The general power $(t, r)$-distance between two points $p=\left(p_{1}, p_{2}, \ldots, p_{n}\right)$ and $\mathrm{q}=\left(\mathrm{q}_{1}, \mathrm{q}_{2}, \ldots, \mathrm{q}_{\mathrm{n}}\right)$ is given by the following:

$$
\text { Power (t, r)-distance }{ }_{p q}=\left(\sum_{\mathrm{k}=1}^{n}\left|\mathrm{p}_{\mathrm{k}}-\mathrm{q}_{\mathrm{k}}\right|^{t}\right)^{\frac{1}{r}}
$$

The special case where $(t, r)=(2,1)$ is called the 'squared Euclidean distance':

$$
\text { Squared Euclidean distance }{ }_{p q}=\left(\sum_{\mathrm{k}=1}^{n}\left|\mathrm{p}_{\mathrm{k}}-\mathrm{q}_{\mathrm{k}}\right|^{2}\right) \text {. }
$$


Therefore, the distance measured by the KSI corresponds to one-quarter of the standardized 'squared Euclidean distance'.

$$
K S I_{i j}=\frac{\text { squared Euclidean distance }(\text { Standardized })_{i j}}{4} .
$$

Kogut and Singh (1988) do not explain why they advocate using a squared function of cultural distance instead of cultural distance. We cannot ascertain a valid reason for this approach rather than assuming that they have erroneously arrived at a squared function of distance as a result of mistakenly taking the average of the 'sum of the squared differences' rather than taking the square root of the 'sum of the squared differences'. Although $\mathrm{x}^{2}$ (squared distance) is positively correlated with $\mathrm{x}$ (distance) when $\mathrm{x}$ is non-negative, it is not appropriate to use the wrong one (squared distance), especially if the correct one (distance) is readily available and using the wrong one leads to biased/incorrect results. If using $\mathrm{x}^{2}$ instead of $\mathrm{x}$ can be justified based on a correlation between $\mathrm{x}^{2}$ and $\mathrm{x}$, then the use of any quadratic function of $\mathrm{x}$ or even any $n$ th-degree polynomial of $\mathrm{x}$ with positive parameters can be justified based on such a correlation. Additionally, the correlation between $\mathrm{x}$ and $\mathrm{x}^{2}$ depends on the exact distribution of the sample, and therefore, the bias introduced by such a transformation will depend on the distribution of the sample. Most importantly, $x$ and $\mathrm{x}^{2}$ have a non-linear correlation, i.e. both variables increase monotonically but not at the same rate (so the ratio of change between the two is not constant). We will discuss the implications of this in section four.

Applied mathematicians use the squared Euclidean distance to place progressively greater weight on objects that are farther apart (Deza and Deza 2016). The squared Euclidean distance is not a distance (metric) function, and it does not satisfy the triangular inequality condition, a critical condition that distance functions need to satisfy (Cuypers et al. 2018; Deza and Deza 2016). To satisfy the triangular inequality condition, for all $\mathrm{x}, \mathrm{y}$, and $\mathrm{z}$ in the distance space, the following inequality should be satisfied:

$$
d(x, y) \leq d(x, z)+d(z, y) .
$$

The triangular inequality condition requires that the distance between two points ( $\mathrm{x}$ and $\mathrm{y}$ ) be the shortest distance along any path. However, the KSI violates this condition. In the simple illustration shown in Fig. 2, the distance between $\mathrm{A}$ and $\mathrm{B}$ should be less than (or equal to) the sum of the distances between $\mathrm{A}$ and $\mathrm{C}$ and between $\mathrm{C}$ and $\mathrm{B}$.

$$
\begin{aligned}
d(A, B) & \leq d(A, C)+d(C, B) \\
8 & \leq 5+5 .
\end{aligned}
$$

However, in the case of the squared Euclidean distances calculated by the KSI;

$$
\begin{aligned}
& K S I(A, B)=d(A, B)^{2} / 4=16 \\
& K S I(A, C)=d(A, C)^{2} / 4=6.25 \\
& K S I(C, B)=d(C, B)^{2} / 4=6.25 \\
& \text { Therefore, } \quad K S I(A, B) \geq K S I(A, C)+K S I(C, B) .
\end{aligned}
$$




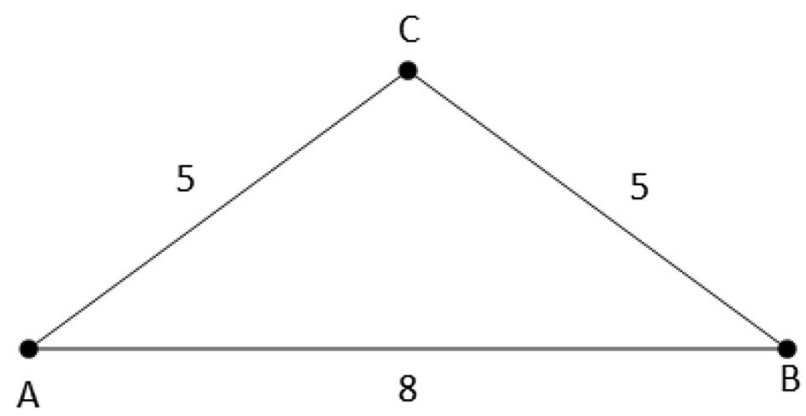

Fig. 2 An example to illustrate how the squared Euclidean distance violates triangular inequality

Thus, the KSI does violate the condition of triangular inequality. We will explain the implications of this issue for international business research below.

\section{Estimating Cultural Distance Using the KSI and Euclidean Distance}

\subsection{A Simple Illustration Based on Three Countries}

To illustrate the issue of exaggerating large distances over small distances, we take a simple example where three countries differ in one cultural dimension but are similar in the other three cultural dimensions. For example, Peru, Turkey, and France differ in their individualism dimension but are similar in the other three dimensions (power distance, masculinity, and uncertainty avoidance).

For simplicity, we assume the differences in power distance, masculinity, and uncertainty avoidance among these three countries to be zero. Then, as depicted in Fig. 3a, the difference in the level of individualism is 21 between Peru and Turkey, 34 between Turkey and France, and 55 between Peru and France. First, we use the KSI to calculate the cultural distances between these three countries (again assuming the differences in other three dimensions are zero) (see Fig. 3b) and compare the KSI with the respective distances in the relevant scores for individualism. Although the distance between Turkey and France (34) is 1.62 times greater than the distance between Peru and Turkey (21), the KSI indicates that the distance between Turkey and France (0.5) is 2.63 times greater than the distance between Peru and Turkey (0.191) (see Fig. 3b). Similarly, although the distance in the values for individualism between Peru and France (55) is 2.62 times greater than the distance between Peru and Turkey (21), the KSI indicates that the distance between Peru and France (1.310) is 6.83 times greater than the distance between Peru and Turkey (0.191) (see Fig. 3b). We selected these three countries for the illustration so that the differences among the countries in the other three cultural dimensions could be kept at zero. However, the inflation of the cultural distance associated with the KSI will be much greater for other country pairs in which the cultural gap is much larger because the KSI inflates these ratios exponentially from $x: 1$ to $x^{2}: 1$. For example, if the difference on a particular dimension between countries in a pair is ten times greater than 

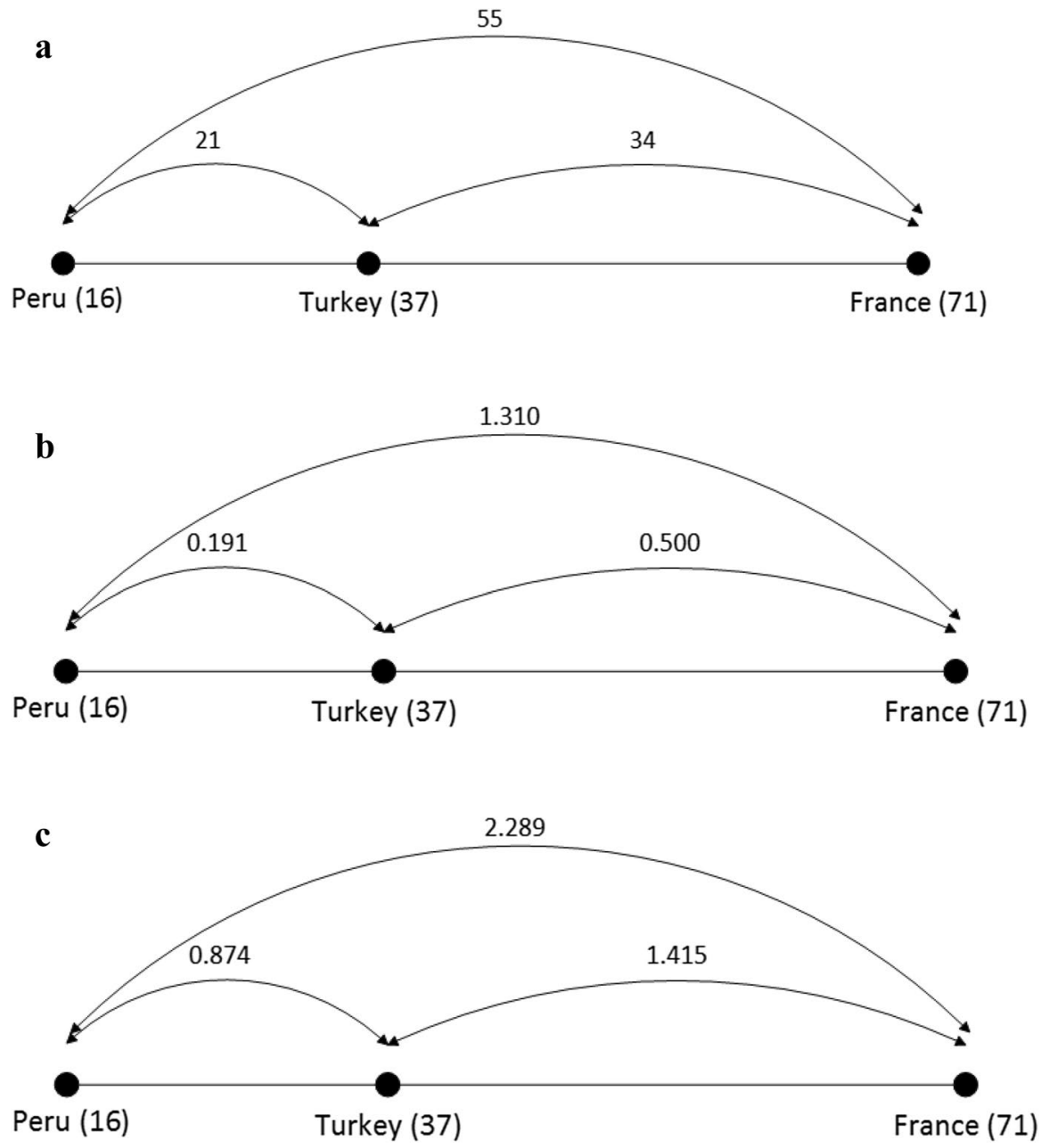

Fig. 3 a Cultural distance based on Hofstede's individualism dimension. b Cultural distances based on the KSI. For ease of illustration/comparison, the differences in the other three dimensions (power distance, masculinity and uncertainty avoidance) between these three countries are assumed to be zero. The cultural distances based on the KSI (considering the all four dimensions) for the country pairs are 0.200 , 0.506 and 1.319 , which are very close to the respective approximated values of $0.191,0.500$ and 1.310 based on the individualism dimension only. c Cultural distance based on Eq. 4b (the Euclidean distance after standardization). As in $\mathbf{b}$, the differences in the other three dimensions of culture between these three countries are assumed to be zero. The Euclidean distance (considering all four dimensions) for the country pairs are $0.896,1.422$ and 2.297 , which are very close to the respective approximated values of $0.874,1.415$ and 2.289 based on the individualism dimension only

the difference on the same dimension between countries in a different pair, then the KSI will inflate this 10:1 ratio to a 100:1 ratio. Thus, when comparing the difference in individualism between the US and Panama (80) with the difference in 
individualism between Japan and Brazil (8), the KSI would state that the difference in individualism between the former is 100 times greater than that between the latter.

In Fig. 3c, we apply Eq. 4b, which is the correct, standardized Euclidean distance formula, to our example. The distance between Turkey and France (1.415) is 1.62 times greater than the distance between Peru and Turkey (0.874). The distance between Peru and France (2.289) is 2.62 times greater than that between Peru and Turkey (0.874). These proportions are exactly the same as the proportions of the distances taken from the Hofstede values (Fig. 3a).

\subsection{KSI vs. Euclidean Distance: Re-analysing the Hofstede Data}

We estimated the KSI and the standardized Euclidean distance for all country pairs for which there are data on Hofstede's four cultural dimensions of power distance, uncertainty avoidance, individualism, and masculinity. Our dataset included 68 countries and 2278 country pairs. ${ }^{1}$ In Fig. 4, we plot the values of the KSI against those of the standardized Euclidean distance. We also plot the standardized Euclidean distance (shown on the diagonal) to graphically illustrate the deviation of the KSI from the standardized Euclidean distance. We can clearly see that the KSI transforms the Euclidean distance to an exponential form because it builds on the squared and not the linear function of distance. Thus, Fig. 4a shows that the KSI transformation places progressively greater weight on the distances as they increase (as shown mathematically in the previous section). The ratio of the KSI to the standardized Euclidean distance increases from 0.07 to 1.5 when the standardized Euclidean distance increases from its lowest value (0.27) to its highest value (6.01). Thus, the KSI places a progressively greater weight (starting from 0.07 and increasing to 1.5) as distance increases. Kogut and Singh (1988) do not provide a theoretical rationale for why this should be the case. To show the significance of this bias in the KSI, we compare this bias at the lowest and highest points of the standardized Euclidean distance. The KSI transforms the lowest distance $(0.27)$ to $0.018(0.27 \times 0.07)$ and the highest distance $(6.01)$ to $9.02(6.01 \times 1.5)$. The highest value of the standardized Euclidean distance is approximately $22(6.01 / 0.27)$ times greater than the smallest distance. However, after the transformation in the KSI, the highest distance becomes approximately $500(9.02 / 0.018)$ times greater than the smallest distance. These results are similar when using the GLOBE data. With the GLOBE data, the highest value of the standardized Euclidean distance is approximately nine times greater than the smallest distance. However, after the transformation in the KSI, the highest distance becomes approximately 80 times greater than the smallest distance.

\footnotetext{
1 We also estimated the KSI and the standardized Euclidean distance based on the nine cultural dimensions reported in the GLOBE study (assertiveness, institutional collectivism, in-group collectivism, future orientation, gender egalitarianism, humane orientation, performance orientation, power distance, and uncertainty avoidance). We follow prior research and focus on the 'practices' indices of these nine dimensions (Schwens et al. 2011). This dataset included 54 countries and 1431 country pairs. Results/ conclusions are similar to those based on the Hofstede data. This dataset is available from the authors.
} 


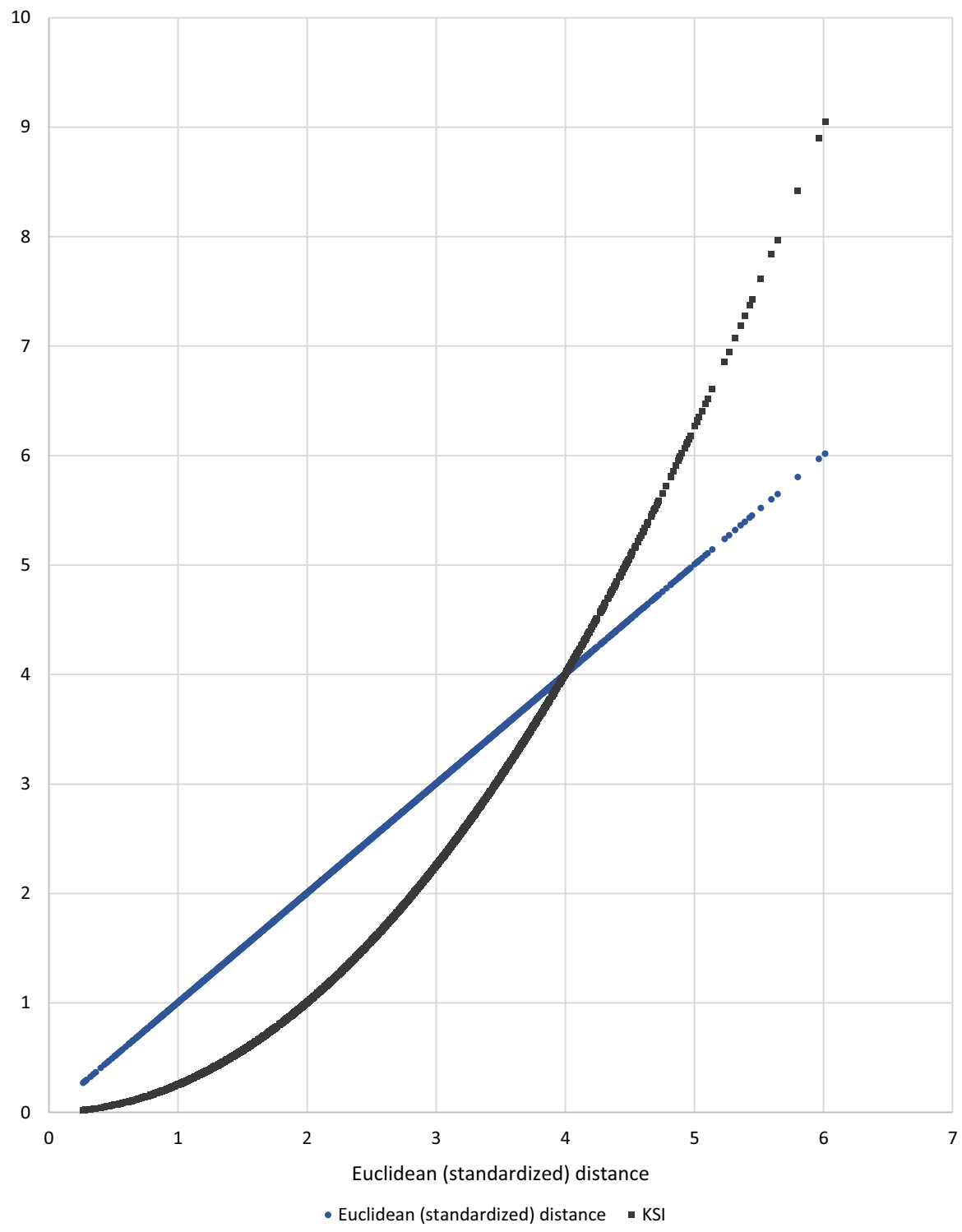

Fig. 4 Euclidean (standardized) distance vs. KSI distance based on Hofstede's cultural measures

\section{Consequences of Using the KSI}

\subsection{Exaggerating Large Distances Over Small Distance}

As shown above, the KSI progressively amplifies the magnitude of distances as they increase. This amplification has important consequences for studies using the KSI. First, the majority of prior studies using the KSI have used it to measure 
cultural distance, which they expect to explain certain outcome variables, including for example, international diversification and entry mode choices (Morschett et al. 2010; Tihanyi et al. 2005; Zhao et al. 2004). Due to the particular nature of the KSI, these studies do not empirically test the effects of cultural distance. Instead, these studies empirically test the effects of squared cultural distance. However, this does not imply that existing studies that found a significant positive or negative effect of cultural distance have in fact found a non-linear effect because they have only included the squared term of cultural distance. To establish a quadratic effect of cultural distance on a particular outcome variable, these studies would have had to include both the second-order term (cultural distance squared) and the first-order term (cultural distance). Simply including the squared term leads to a misspecification as one would try to fit data to an $a x^{2}+c$ function rather than to the standard $a x^{2}+b x+c$ function. The $a x^{2}+c$ function does not provide a U-curve or an inverted $\mathrm{U}$-curve when the range of $\mathrm{x}$ (cultural distance) is positive. This is because the vertex of the parabola (the lowest point on a U-curve or the highest point on an inverted U-curve) lies on the y-axis, thereby imposing half a parabola instead of the full parabola on the data (the vertex is given by $x=-2 b / a$, and, since we are forcing $b=0$, the vertex has the $\mathrm{x}$-coordinate of $x=0$ ). This is a strong assumption (Cohen et al. 2003; Haans et al. 2016). When testing for curvilinear effects, all lower order terms must be included for higher order terms to have a meaning, i.e. the quadratic equation should contain the linear term for the second-order term to have a meaning (Cohen et al. 2003). If the linear term of cultural distance is not included in the quadratic equation, the regression coefficient for the squared cultural distance would confound the linear and the quadratic variance of cultural distance (Cohen et al. 2003). Thus, the empirical results of studies using the KSI to test the effects of cultural distance on different outcome variables are likely to be misleading. If a study proposes a linear effect of cultural distance and then finds no support for this hypothesis (i.e. the results show that the KSI is not statistically significant), we cannot rule out the existence of a linear effect as the study has in fact not tested for the existence of linear effect of cultural distance. If instead a study finds that the KSI is statistically significant, however, this does not mean that they have found support for a linear effect of cultural distance either. This is because a statistically significant KSI may mean that cultural distance has a monotonically increasing/decreasing non-linear effect. Theoretically, a non-linear effect of cultural distance may indicate that a negative effect of culture (e.g. increase in transaction costs) may become more pronounced with increasing cultural distance; or that a positive effect of culture, due to arbitrage effects, for instance, may grow exponentially with increasing cultural distance. However, it is not possible to infer the actual nature of non-linear effects of cultural distance and 'reinterpret' the findings of prior studies into the effects of cultural distance using the KSI because these studies have not included the first-order term (cultural distance) in their model specification(Cohen et al. 2003; Haans et al. 2016). In any case, the researchers using the KSI to capture the effects of cultural distance have not theoretically proposed a monotonically increasing/decreasing nonlinear effect in the first place.

Second, several studies have argued for curvilinear effects of cultural distance, suggesting a U-curve or an inverted $U$ curve shape relationship between cultural 
distance and the dependent variable. Theoretically, the most common approach to conceptualize a U-curve or an inverted $U$ curve shape relationship is to jointly consider two countervailing forces (Haans et al. 2016), such as jointly considering costs and benefits of cultural distance. For example, Shin et al. (2017) argue for a U-curve-shaped relationship between cultural distance and the proportion of expatriate parent country nationals in foreign subsidiaries. Likewise, Malhotra et al. (2011) expect a U-curve-shaped relationship between cultural distance and equity participation in cross-border acquisitions. In both of these studies the initially negative effect of cultural distance on the respective dependent variable was theorised to weaken and turn positive at higher levels of cultural distance. To test their respective hypotheses, these studies include both the first-order KSI and the second-order KSI. However, due to the quadratic nature of the KSI, these studies essentially include the second-order term and the fourth-order term of cultural distance. Thus, instead of fitting a quadratic polynomial (a polynomial of degree 2), these studies have inadvertently fitted a specific form of a quartic polynomial (a polynomial of degree 4) to their data. Even if the intention would have been to fit a quartic polynomial $\left(a x^{4}+b x^{3}+c x^{2}+d x+e\right)$, the inclusion of only a second-order term $\left(x^{2}\right)$ and a fourth-order term $\left(x^{4}\right)$ forces $\mathrm{b}=0$ and $\mathrm{d}=0$ and thus constitutes a misspecification (Cohen et al. 2003). As mentioned above, when testing for curvilinear effects, all lower order terms must be included for higher order terms to have a meaning (Cohen et al. 2003). For example, Malhotra et al. (2011) find 'empirical support' for the suggested U-curve relationship between cultural distance and equity participation in cross-border acquisitions. However, because they have used the KSI, their results are more likely to indicate a relationship in the shape of a ' $W$ ' rather than that of a ' $U$ '.

Third, the particular specification of the KSI also affects studies that test a moderating effect of cultural distance. A moderating effect of cultural distance is theorised when the effect of an explanatory variable on the dependent variable is expected to increase/ decrease with cultural distance. Such a moderating effect can take a linear or a non-linear form but the most common approach is to propose a linear moderating effect of cultural distance. For example, prior research has tested the moderating effect of cultural distance on the relationship between foreign ownership and the extent of control (Puck et al. 2016) or between the use of expatriates and the performance of overseas subsidiaries (Colakoglu and Caligiuri 2008). Because of the use of a quadratic function of distance in the KSI, these studies are in fact testing the moderating effect of squared cultural distance rather than the moderating effect of cultural distance. Unfortunately, the results of these studies cannot be 'transformed back' into the non-quadratic moderating effects of cultural distance that these studies hypothesized about and that they set out to test. If a study proposing a moderating effect has found no support for their hypothesis, we cannot rule out the existence of a linear moderating effect because the study has not in fact tested for the existence of a linear moderating effect of cultural distance. If, on the other hand, the study finds a significant moderating effect of cultural distance, this would not necessarily mean that there is empirical support for a linear moderating effect of cultural distance. Instead, due to the misspecification of the KSI such a finding may mean there is a monotonically increasing/decreasing non-linear moderating effect (i.e. the moderating effect of cultural distance monotonically increases/decreases as cultural distance increases). As in the case of the direct effect of cultural distance, however, 
the specific nature of a non-linear moderating effect cannot be established, because the first-order moderating term (i.e. the interaction term with cultural distance) is in fact not included in the specification. Similarly, these studies would not have theorized about a non-linear moderating effect of cultural distance in their study in the first place. As in the case of studies testing a direct effect of cultural distance, it is thus not possible to interpret the results of these studies in a meaningful way.

Fourth, the nature of the KSI also leads to wrong or misleading results in studies that take the average or the total of cultural distance across multiple dyads (see for example, Beamish and Kachra 2004; Hutzschenreuter and Voll 2008; Hutzschenreuter et al. 2011; Mohr et al. 2016) because the KSI results in averages and sums being computed on the basis of squared cultural distance values. For example, two firms from different countries may each invest in three host countries A, B, C, where the cultural distances between the firms' home countries and the three host countries are 2, 2, and 2 for the first firm and 0, 1, and 5 for the second firm. A researcher taking the average or the total cultural distance would assume that these are the same for both firms, as the average is 2 and the total is 6 in both cases. However, if one uses the KSI, the average or total cultural distances faced by these two firms would differ. By squaring these distances and taking the arithmetic mean, the KSI would transform these cultural distance profiles to 1,1 , and 1 ; and $0,0.25$, and 6.25 , respectively. Based on the KSI, the second firm's total/average cultural distance would be more than twice as great as the first firm's cultural distance. Therefore, the KSI leads to misleading values when researchers compute total or average cultural distance across multiple dyads.

Finally, as a result of the misspecification of the KSI, the usefulness of the findings of existing meta-analyses of the linear, non-linear, moderating and other effects of cultural distance (Beugelsdijk et al. 2018b; Magnusson et al. 2008; Tihanyi et al. 2005; Zhao et al. 2004) will vary with the degree to which the included individual studies have used the KSI.

\subsection{Implications of Violating Triangular Inequality}

The KSI's violation of the triangular inequality condition has consequences particularly for studies that investigate the role of cultural distance for sequential foreign expansion. For instance, Hutzschenreuter et al. (2014) have studied the effects of added psychic distance, i.e. the additional cultural distance from one investment (or existing investment base) to another on MNE performance. Assuming the triangular inequality condition, some of these studies (e.g., Gabriel and Gripsrud 1992) calculate the added cultural distance as $\mathrm{CD}_{\mathrm{n}+1}-\mathrm{CD}_{\mathrm{n}}$, where $\mathrm{CD}_{\mathrm{n}}$ is the cultural distance from the home country to the location of investment number n. Such studies make the following assumption that should be valid in a distance function:

When $C D_{n}=x$ and $C D_{n+1}=x+\delta \mathrm{x}$, Then $C D_{n+1}-C D_{n}=\delta \mathrm{x}$.

However, because the KSI violates the triangular inequality condition, this assumption is no longer valid. If we denote the KSI transformation as KSI, then;

$$
\operatorname{KSI}(x+\delta x)-\operatorname{KSI}(x) \neq \operatorname{KSI}(\delta x) .
$$


This can be proven as follows:

$$
\begin{gathered}
\text { Since } K S I(x+\delta x)=\frac{(x+\delta x)^{2}}{4} \\
K S I(x+\delta x)=\frac{x^{2}}{4}+\frac{(\delta x)^{2}}{4}+\frac{x \cdot \delta x}{2} \\
K S I(x+\delta x)=\operatorname{KSI}(x)+K S I(\delta x)+\frac{x \cdot \delta x}{2}
\end{gathered}
$$

Therefore, $\operatorname{KSI}(x+\delta x)-\operatorname{KSI}(x)=\operatorname{KSI}(\delta x)+\frac{x \cdot \delta x}{2}$.

The error $\left(\frac{x \cdot \delta x}{2}\right)$ introduced by the KSI transformation increases with $x$. To illustrate this effect, we can take an example where $C D_{n}=2$ and $C D_{n+1}=4$. If we use the KSI to calculate the cultural distance (as is the case in Gabriel and Gripsrud 1992), then $\mathrm{CD}_{\mathrm{n}}$ and $\mathrm{CD}_{\mathrm{n}+1}$ transform to $1\left(=2^{2} / 4\right)$ and $4\left(=4^{2} / 4\right)$, respectively. Although the firm's second investment in this case occurs in a country that is twice as far from the home country than from the country it expanded to in its first investment, the KSI suggests that the country of the firm's second investment is four times the distance of the country it entered in the first investment. The KSI substantially inflates even a small increment, especially if the $n$th investment is already culturally distant from the home country, because the error term is a function of $x$, which is the distance to the existing investments. Some studies (see, for instance, Hutzschenreuter et al. 2011) have calculated the added cultural distance as the cultural distance between the $n$th location (or existing investment base) and the $(n+1)$ th location. This approach is better than taking the difference between $\mathrm{CD}_{\mathrm{n}}$ and $\mathrm{CD}_{\mathrm{n}+1}$. However, if these studies use both added cultural distance $(\delta x)$ and existing cultural distance $(x)$ (as in the case of Hutzschenreuter et al. 2011), then $\delta x$ will be underestimated when $\mathrm{x}$ is larger.

The KSI's violation of the triangular inequality also leads to a distortion of distances in the 'cultural space' with detrimental consequences particularly for studies that investigate the role of cultural distance in the context of multilateral, rather than bilateral, relationships among countries, firms, or individuals. Scholars have examined, for instance, the cultural distances among the partners in IJVs with three or more partners from different countries (Mohr et al. 2016). To illustrate this, as shown in Fig. 5, South Africa is equally distant from Slovakia and Sweden. However, because the KSI violates the triangular inequality condition, the location of the three countries in cultural space no longer makes any sense when researchers use the KSI to compute the cultural distances. Someone comparing the KSI between Slovakia and Sweden (9.04) with the KSI between Slovakia and South Africa (2.37) will conclude that South Africa is culturally closer to Slovakia than Sweden. In contrast, someone comparing the KSI between Sweden and Slovakia (9.04) and the KSI between Sweden and South Africa (2.67) will conclude that South Africa is culturally closer to Sweden than Slovakia. Thus, the KSI is unable to provide meaningful 


\section{Euclidean distance (standardized)}

Slovakia

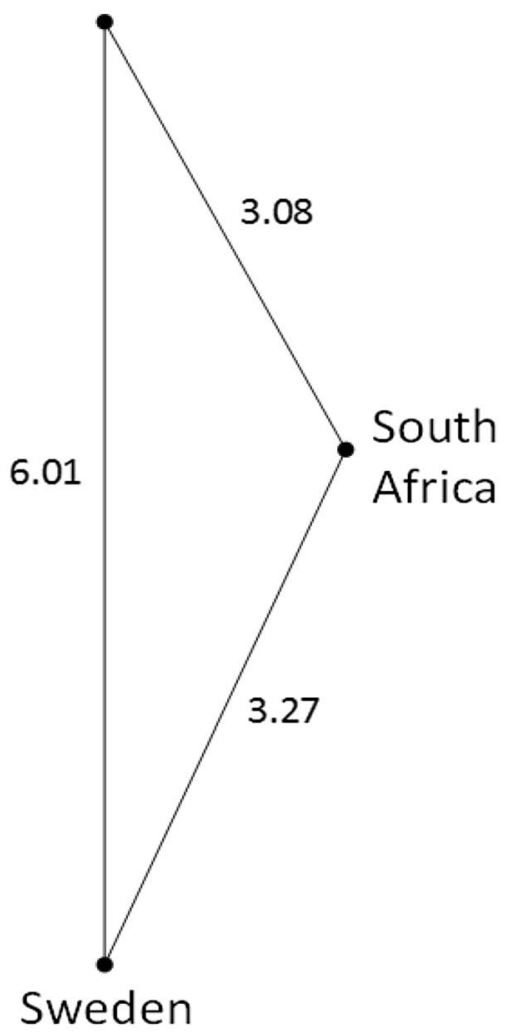

$\underline{\mathrm{KSI}}$

Slovakia

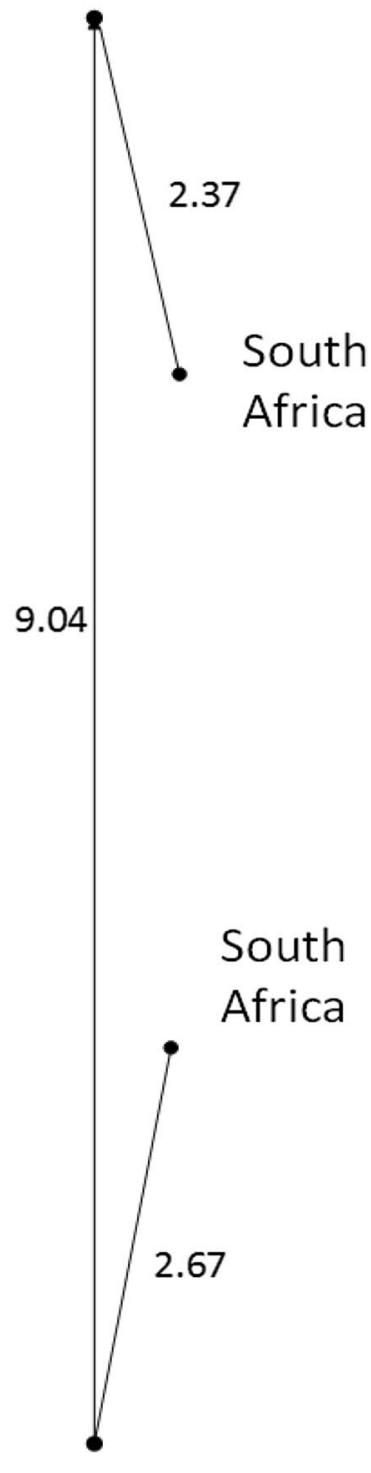

Sweden

Fig. 5 Distortion of distances in the 'cultural space' arising from the KSI's violation of the triangular inequality 
insights into the cultural distance between these three countries. Such distortions arising from the KSI's violation of the triangular inequality condition has consequences particularly for studies that investigate the role of cultural distance between three (or more) locations or actors, such as in multi-partner IJVs or in acquisitions where the acquirer, seller and the acquisition target are based in different countries.

\section{Conclusion}

The KSI remains one of, if not the single, most widely used constructs in international business given the central role of cultural (and other) distance in our discipline. We show mathematically and empirically that the KSI is incorrectly specified. We explain the problems associated with using the KSI, which calculates 'squared cultural distance' and does not satisfy triangular inequality as one of the key conditions for distance functions. We also examine the serious implications of using the KSI in different areas of international business research. In so doing, our study contributes to resolving the confusion among scholars regarding the distinction between the KSI and Euclidean distance. The terms KSI and Euclidean distance continue to be used interchangeably by both, authors that have used the KSI and the (small number of) scholars that have used the Euclidean distance. Our paper provides compelling reasons for avoiding the use of the KSI in future research.

On a more general level, a small number of scholars has started questioning the relevance of and the assumptions underlying the concept of cultural distance (e.g., Harzing and Pudelko 2016; López-Duarte et al. 2016; Maseland et al. 2018; Shenkar 2001; Tung and Verbeke 2010). One of the most prominent examples is Shenkar (2001)'s JIBS decade award winning article, in which he challenges the implicit assumptions and the theoretical and methodological properties of the concept of cultural distance. However, many scholars' scepticism with regard to the concept of cultural distance results from the inconsistent results in the prior research employing the KSI and the apparent weak explanatory power of the KSI (Shenkar 2001, 2012; Tung and Verbeke 2010). For example, Shenkar (2001) shows that the prior empirical evidence related to the impact of cultural distance on (1) launch/sequence of foreign investment, (2) entry mode, and (3) affiliate performance is all inconclusive and uses this weak explanatory power of cultural distance as measured by the KSI as one of the main reason for his scepticism about the KSI. Our intention is not to question these criticisms of the relevance and the assumptions underlying the concept of cultural distance. Instead, we believe that the misspecification of the KSI has contributed to those inconsistencies in prior findings and weak explanatory power for the concept of cultural distance. Interestingly, one of the main criticism levelled against the KSI (Shenkar 2001; Tung and Verbeke 2010) is the assumption of linearity in the studies employing cultural distance construct, i.e. assuming that there is a linear association between cultural distance and the selected dependent variable. For example, assuming that when the cultural distance measure increases, the likelihood of MNEs choosing a joint venture over a wholly owned subsidiary (or vice versa), should also change proportionally. The impact of cultural distance may well not 
be linear as suggested in most prior research on the effects of cultural distance. Instead, cultural distance may potentially have non-linear effects. For instance, the positive or negative effects of cultural distance may grow exponentially with growing cultural distance, or there may be one or more threshold levels at which the nature of the effect of cultural distance changes. Future studies should explore the theoretical rationales for such potential non-linear effects of cultural distance and test these effects using the correct form of the Euclidean distance formula and the correct form of model specification (i.e. correct form of polynomial).

Our conclusion is not very encouraging in terms of the validity of the accumulated research using the KSI to investigate the effects of cultural distance. We are not able to provide a solution that would allow a transformation or reinterpretation of past empirical research that has used the KSI. However, we do believe that future studies should use the correct form of the Euclidean distance formula to investigate the effects of cultural (and other) distance in firm internationalization. Since much of what we know about these effects rests on the empirical results obtained from using the KSI, replicating previous studies using the correct index for measuring cultural distance would be central for our understanding of the effects of cultural distance. We strongly caution against the use of the KSI to measure cultural or other distances between countries or firms and recommend employing alternative measures.

One limitation of our paper is that we exclusively focus on and remedy the misspecification of the KSI. First, partially because of the criticism levelled against the concept of cultural distance, research has started using alternative indices for measuring distance (including, cultural distance). In particular, Berry et al. (2010) have proposed that researchers should use the Mahalanobis distance, which takes accounts for the correlation between the dimensions. The Mahalanobis distance is equivalent to the Euclidean distance computed with the standardized values of the principal components (De Maesschalck et al. 2000). Whether the Mahalanobis distance is superior to the Euclidean distance depends on whether researchers prefer to use the original dimensions or the principle components of the original dimensions and on the context of the application. The Euclidean distance may also be preferred over the Mahalanobis distance, because (a) the Euclidean distance is easier to compute and interpret than the Mahalanobis distance; because (b) the greater difficulties of computing the variance-covariance matrix required by the Mahalanobis distance measure, when the variance-covariance matrix is singular or nearly singular and cannot be inverted; and because (c) the Euclidean distance can handle overdetermination (i.e., for the calculation of the variance-covariance matrix the number of objects in the data set has to be larger than the number of variables) (De Maesschalck et al. 2000). Comparing the incorrectly specified KSI with the Mahalanobis distance, Kandogan (2012) observes that they lead to different estimates. As we have illustrated in our paper, this is because, in contrast to the Mahalanobis distance, the KSI is a squared distance measure. Rather than highlighting the misspecification of the KSI, however, Kandogan (2012) seems to use the KSI as benchmark and suggest modifying the Mahalanobis distance measure to make it comparable with the KSI: "The modified version would basically square the original Mahalanobis distance and divide it by the number of dimensions so that it is comparable to the Kogut and Singh measures" (Kandogan 2012, p. 199). We do not recommend this approach as 
the resulting 'squared Mahalanobis' distance measure has the same empirical and theoretical shortcomings as the KSI that we discuss in our paper.

Second, although the KSI has been and continues to be the most prominent way to measure cultural distance in international business research, we do not engage with, let alone provide solutions for the various other concerns that have been raised with regard to the use and measurement of the cultural distance concept in international business research. Our contribution thus complements suggestions on improving various other facets of research on cultural distance (e.g., Brouthers et al. 2016). The concept of cultural distance continues to play a central role in various areas of research in international business and management and the use of a correct measure of this concept is thus of great importance.

Acknowledgements Open access funding provided by Vienna University of Economics and Business (WU).

Open Access This article is distributed under the terms of the Creative Commons Attribution 4.0 International License (http://creativecommons.org/licenses/by/4.0/), which permits unrestricted use, distribution, and reproduction in any medium, provided you give appropriate credit to the original author(s) and the source, provide a link to the Creative Commons license, and indicate if changes were made.

\section{References}

Anton, H., \& Rorres, C. (2014). Elementary linear algebra: Applications version. Hoboken: Wiley.

Beamish, P. W., \& Kachra, A. (2004). Number of partners and JV performance. Journal of World Business, 39(2), 107-120.

Berry, H., Guillén, M. F., \& Nan, Z. (2010). An institutional approach to cross-national distance. Journal of International Business Studies, 41(9), 1460-1480.

Beugelsdijk, S., Ambos, B., \& Nell, P. C. (2018a). Conceptualizing and measuring distance in international business research: Recurring questions and best practice guidelines. Journal of International Business Studies, 49(9), 1113-1137.

Beugelsdijk, S., Kostova, T., Kunst, V. E., Spadafora, E., \& van Essen, M. (2018b). Cultural distance and firm internationalization: A meta-analytical review and theoretical implications. Journal of Management, 44(1), 89-130.

Brouthers, K. D., \& Brouthers, L. E. (2001). Explaining the national cultural distance paradox. Journal of International Business Studies, 32(1), 177-189.

Brouthers, L. E., Marshall, V. B., \& Keig, D. L. (2016). Solving the single-country sample problem in cultural distance studies. Journal of International Business Studies, 47(4), 471-479.

Chen, G., Kirkman, B. L., Kim, K., Farh, C. I. C., \& Tangirala, S. (2010). When does cross-cultural motivation enhance expatriate effectiveness? A multilevel investigation of the moderating roles of subsidiary support and cultural distance. Academy of Management Journal, 53(5), 1110-1130.

Cohen, J., Cohen, P., West, S. G., \& Aiken, L. S. (2003). Applied multiple regression/correlation analysis for the behavioral sciences. London: Taylor and Francis.

Colakoglu, S., \& Caligiuri, P. (2008). Cultural distance, expatriate staffing and subsidiary performance: The case of US subsidiaries of multinational corporations. The International Journal of Human Resource Management, 19(2), 223-239.

Cuypers, I. R. P., Ertug, G., Heugens, P. P. M. A. R., Kogut, B., \& Zou, T. (2018). The making of a construct: Lessons from 30 years of the Kogut and Singh cultural distance index. Journal of International Business Studies, 49(9), 1138-1153.

De Maesschalck, R., Jouan-Rimbaud, D., \& Massart, D. L. (2000). The Mahalanobis distance. Chemometrics and Intelligent Laboratory Systems, 50(1), 1-18.

Deza, M. M., \& Deza, E. (2016). Encyclopedia of distances. Berlin, Heidelberg: Springer. 
Evans, J., \& Mavondo, F. T. (2002). Psychic distance and organizational performance: An empirical examination of international retailing operations. Journal of International Business Studies, 33(3), $515-532$.

Gabriel, R. G. B., \& Gripsrud, G. (1992). The expansion of foreign direct investments: Discrete rational location choices or a cultural learning process? Journal of International Business Studies, 23(3), 461-476.

Gerschewski, S. (2013). Improving on the Kogut and Singh metric of psychic distance. Multinational Business Review, 21(3), 257-268.

Haans, R. F. J., Pieters, C., \& He, Z.-L. (2016). Thinking about U: Theorizing and testing U- and inverted U-shaped relationships in strategy research. Strategic Management Journal, 37(7), 1177-1195.

Harzing, A.-W., \& Pudelko, M. (2016). Do we need to distance ourselves from the distance concept? Why home and host country context might matter more than (cultural) distance. Management International Review, 56(1), 1-34.

Hofstede, G. (1980). Culture's consequences: International differences in work-related values. Beverly Hills, London: Sage Publications.

Hutzschenreuter, T., Kleindienst, I., \& Lange, S. (2014). Added psychic distance stimuli and MNE performance: Performance effects of added cultural, governance, geographic, and economic distance in MNEs' international expansion. Journal of International Management, 20(1), 38-54.

Hutzschenreuter, T., \& Voll, J. C. (2008). Performance effects of "added cultural distance" in the path of international expansion: The case of German multinational enterprises. Journal of International Business Studies, 39(1), 53-70.

Hutzschenreuter, T., Voll, J. C., \& Verbeke, A. (2011). The impact of added cultural distance and cultural diversity on international expansion patterns: A Penrosean perspective. Journal of Management Studies, 48(2), 305-329.

Kandogan, Y. (2012). An improvement to Kogut and Singh measure of cultural distance considering the relationship among different dimensions of culture. Research in International Business and Finance, 26(2), 196-203.

Kirkman, B. L., Lowe, K. B., \& Gibson, C. B. (2006). A quarter century of Culture's Consequences: A review of empirical research incorporating Hofstede's cultural values framework. Journal of International Business Studies, 37(3), 285-320.

Koch, P. T., Koch, B., Menon, T., \& Shenkar, O. (2016). Cultural friction in leadership beliefs and foreign-invested enterprise survival. Journal of International Business Studies, 47(4), 453-470.

Kogut, B., \& Singh, H. (1988). The effect of national culture on the choice of entry mode. Journal of International Business Studies, 19(3), 411-432.

Kraimer, M. L., Shaffer, M. A., Harrison, D. A., \& Ren, H. (2012). No place like home? An identity strain perspective on repatriate turnover. Academy of Management Journal, 55(2), 399-420.

Liu, Y., Ge, Y., Hu, Z., \& Wang, S. (2018). Culture and capital flows-Exploring the spatial differentiation of China's OFDI. China Economic Review (1043951X), 48, $27-45$.

López-Duarte, C., Vidal-Suárez, M. M., \& González-Díaz, B. (2016). International business and national culture: A literature review and research agenda. International Journal of Management Reviews, 18(4), 397-416.

Magnusson, P., Baack, D. W., Zdravkovic, S., Staub, K. M., \& Amine, L. S. (2008). Meta-analysis of cultural differences: Another slice at the apple. International Business Review, 17(5), 520-532.

Malhotra, S., Sivakumar, K., \& Zhu, P. (2011). Curvilinear relationship between cultural distance and equity participation: An empirical analysis of cross-border acquisitions. Journal of International Management, 17(4), 316-332.

Manev, I. M., \& Stevenson, W. B. (2001). Nationality, cultural distance, and expatriate status: Effects on the managerial network in a multinational enterprise. Journal of International Business Studies, 32(2), 285-303.

Maseland, R., Dow, D., \& Steel, P. (2018). The Kogut and Singh national cultural distance index: Time to start using it as a springboard rather than a crutch. Journal of International Business Studies, 49(9), 1154-1166.

Mohr, A., Wang, C., \& Goerzen, A. (2016). The impact of partner diversity within multiparty international joint ventures. International Business Review, 25(4), 883-894.

Morosini, P., Shane, S., \& Singh, H. (1998). National cultural distance and cross-border acquisition performance. Journal of International Business Studies, 29(1), 137-158. 
Morschett, D., Schramm-Klein, H., \& Swoboda, B. (2010). Decades of research on market entry modes: What do we really know about external antecedents of entry mode choice? Journal of International Management, 16(1), 60-77.

Puck, J., Hödl, M. K., Filatotchev, I., Wolff, H.-G., \& Bader, B. (2016). Ownership mode, cultural distance, and the extent of parent firms' strategic control over subsidiaries in the PRC. Asia Pacific Journal of Management, 33(4), 1075-1105.

Reus, T. H., \& Lamont, B. T. (2009). The double-edged sword of cultural distance in international acquisitions. Journal of International Business Studies, 40(8), 1298-1316.

Salomon, R., \& Wu, Z. (2012). Institutional distance and local isomorphism strategy. Journal of International Business Studies, 43(4), 343-367.

Schwens, C., Eiche, J., \& Kabst, R. (2011). The moderating impact of informal institutional distance and formal institutional risk on SME entry mode choice. Journal of Management Studies, 48(2), $330-351$.

Shenkar, O. (2001). Cultural distance revisited: Towards a more rigorous conceptualization and measurement of cultural differences. Journal of International Business Studies, 32(3), 519-535.

Shenkar, O. (2012). Beyond cultural distance: Switching to a friction lens in the study of cultural differences. Journal of International Business Studies, 43(1), 12-17.

Shenkar, O., Luo, Y., \& Yeheskel, O. (2008). From "distance" to "friction": Substituting metaphors and redirecting intercultural Research. The Academy of Management Review, 33(4), 905-923.

Shin, D., Hasse, V. C., \& Schotter, A. P. J. (2017). Multinational enterprises within cultural space and place: Integrating cultural distance and tightness-looseness. Academy of Management Journal, 60(3), 904-921.

Tihanyi, L., Griffith, D. A., \& Russell, C. J. (2005). The effect of cultural distance on entry mode choice, international diversification, and MNE performance: A meta-analysis. Journal of International Business Studies, 36(3), 270-283.

Tung, R. L., \& Verbeke, A. (2010). Beyond Hofstede and GLOBE: Improving the quality of cross-cultural research. Journal of International Business Studies, 41(8), 1259-1274.

Varela, O. E., \& Gatlin-Watts, R. (2014). The development of the Global Manager: An empirical study on the role of academic international Sojourns. Academy of Management Learning and Education, 13(2), 187-207.

Yu, L. I. U., \& Maula, M. (2016). Local partnering in foreign ventures: Uncertainty, experimental learning, and syndication in cross-border venture capital investments. Academy of Management Journal, 59(4), 1407-1429.

Zaheer, S., Schomaker, M. S., \& Nachum, L. (2012). Distance without direction: Restoring credibility to a much-loved construct. Journal of International Business Studies, 43(1), 18-27.

Zhao, H., Luo, Y., \& Suh, T. (2004). Transaction cost determinants and ownership-based entry mode choice: A meta-analytical review. Journal of International Business Studies, 35(6), 524-544.

Publisher's Note Springer Nature remains neutral with regard to jurisdictional claims in published maps and institutional affiliations. 